\section{Acidose, respiratorische}

O. Müller-Plathe

Hamburg, Deutschland

\section{Englischer Begriff respiratory acidosis}

Definition Die respiratorische Acidose ist die durch Anreicherung von Kohlendioxid (Hyperkapnie) infolge alveolärer Hypoventilation entstehende Störung des Säure-Basen-Gleichgewichts.

Beschreibung Allen respiratorischen Acidosen gemeinsam sind die Zunahme von $p \mathrm{CO}_{2}$ und die Tendenz zur pH-WertSenkung, die je nach Grad der Kompensation unterschiedlich stark ausgeprägt ist.

Im Falle der akuten respiratorischen Acidose steht lediglich die Pufferung durch die Nicht-Bicarbonatpuffer zur Verfügung, bei der Bicarbonat im Wesentlichen auf Kosten von $\mathrm{Hb}^{-}$gebildet wird.

Laborkonstellation:

- $p \mathrm{CO}_{2}$ mäßig bis stark erhöht, $c \mathrm{HCO}_{3}{ }^{-}$mäßig erhöht $(\leq 32 \mathrm{mmol} / \mathrm{L})$

- Basenabweichung normal, $\mathrm{pH}$ relativ stark gesenkt $(\geq 7,1)$

Bei der chronischen respiratorischen Acidose tritt im Verlauf mehrerer Tage die renale Bicarbonatbildung als Kompensationsvorgang hinzu ( $\triangleright$ Säureausscheidung, renale).

Laborkonstellation:

- $p \mathrm{CO}_{2}$ mäßig bis stark erhöht, $c \mathrm{HCO}_{3}{ }^{-}$stark erhöht (bis ca. $50 \mathrm{mmol} / \mathrm{L}$ )

- Basenabweichung deutlich erhöht (bis ca. $15 \mathrm{mmol} / \mathrm{L}$ ), $\mathrm{pH}$ nur mäßig gesenkt $(\geq 7,25)$
Die Entscheidung, ob die Störung in normaler Weise kompensiert ist oder nicht, kann mithilfe des Diagramms im Stichwort \ Säure-Basen-Stoffwechsel getroffen werden.

Vorkommen bei folgenden Störungen:

- Atemzentrum: Tumor, Hirnblutung, Enzephalitis, Ischämie, Narkotika, Pickwick-Syndrom

- Peripheres Nervensystem: hohe Querschnittsläsion, doppelseitige Phrenicusparese, Polyneuropathie

- Muskulatur: Myasthenie, Botulismus, Myositis, Muskeldystrophie, hypokaliämische Lähmung, Muskelrelaxantien, Operationstrauma

- Thorax: Deformitäten, Rippenserienfraktur, Pneumothorax

- Atemwege: Fremdkörper, Aspiration, Verschleimung, Tumor, bronchostenotisches Emphysem, Status asthmaticus im Ermüdungsstadium

- Lunge: Pneumonie, Lungenödem, Zystenlunge, Schocklunge, ARDS

- Mechanische Beatmung: Hypoventilation, zu hoher Totraumanteil

Auswirkungen Die respiratorische Acidose, die in der akuten Form mit heftiger Dyspnoe verbunden ist, stellt durch den gleichzeitig bestehenden Sauerstoffmangel stets einen lebensbedrohenden Zustand dar, der sofortiges Handeln erfordert. Es kann zu Tachykardie, Herzrhythmusstörungen und Blutdrucksteigerungen kommen. Weitere Auswirkungen sind Verwirrtheit, Tremor und Steigerung des Liquordrucks, Tendenz zur Hyperkaliämie und Hypochlorämie.

\section{Literatur}

Epstein SK, Singh N (2001) Respiratory acidosis. Respir Care 46(4):366-383 